\title{
Enhancement of mononuclear cell oxidative burst by early post-transfer sera from human patients after unsuccessful embryo transfer
}

\author{
Friedrich E.Maly ${ }^{1}$, Karl Sterzik ${ }^{2}$, David Henderson ${ }^{3}$ \\ and Alain L.de Weck \\ Institute of Clinical Immunology, Inselspital Bern, CH-3010 \\ Switzerland, ${ }^{2}$ Department of Gynaecology, University of UIm and \\ ${ }^{3}$ Biochemical Pharmacology, Schering AG Berlin, FRG \\ 'To whom correspondence should be addressed
}

Early pregnancy sera were earlier shown to modulate $T$ lymphocyte rosetting with sheep erythrocytes. We set out to investigate whether early pregnancy sera also modulate the state of activation of another immunologically relevant cell type, the monocyte. As an index of monocyte activation, we measured phorbol ester-triggered oxidative burst activity by a highly sensitive chemiluminescence method. Contrary to our expectations, incubation of mononuclear cells with sera taken early after embryo transfer from patients with a successfully developing pregnancy had no effect. Unexpectedly, such sera from patients from a control group of patients in whom no pregnancy developed after embryo transfer caused enhancement of mononuclear cell chemiluminescence. Stimulatory activity of these sera appeared between days 4 and 6 and was maximal between days 7 and 9 post embryo transfer. Whether this phenomenon is causally related to, or represents a consequence of the failure of embryo transfer, can currently not be decided.

Key words: embryo transfer failure/monocytes/oxidative burst/serum factors

\section{Introduction}

The concept of circulating serum factors indicative of the state of a pre- or peri-implantation embryo (early pregnancy factor, EPF) was originally developed by Morton et al. from their finding of serum factors able to co-inhibit rosette formation between $\mathrm{T}$ lymphocytes and sheep red blood cells (Morton et al., 1976). Since this original observation, results reported from a number of laboratories led to the conclusion that the signalling systems between embryo and mother are complex in nature and utilize a wide variety of mediators, some of which are also involved in immune reactions. Thus, for example, O'Neill et al. showed that in the mouse, successful fertilization is accompanied by peripheral thrombocytopenia due to the release of a embryoderived platelet-activating factor (O'Neill et al., 1985). More recently, the ovine trophoblast protein OTP-1 was shown to be a member of the interferon super-family and indeed anti-luteolytic activity of interferons can be demonstrated in some species (Imakawa et al., 1987). These and other mediators appear to be largely or wholly involved in the control of luteal function. The presence of large numbers of infiltrating leukocytes at the site of implantation and the necessity for explaining the immunologically privileged nature of the embryo suggest, however, that the implanting embryo may modulate some component(s) of the maternal immune system. In addition to the central role of $\mathrm{T}$ lymphocytes, accessory cells such as monocytes/macrophages participate in the regulation of the immune response. We therefore investigated whether monocyte activity is influenced by serum obtained in early pregnancy, with the additional aim of developing an alternative to the rosette inhibition test for early pregnancy factor (EPF). Depending on their activation state, monocytes/ macrophages produce a great variety of biologically active mediators, ranging from reactive oxygen species such as superoxide and lipid mediators such as prostaglandins to peptide mediators, for example GM-CSF and IL-1. In the present study, we investigated whether sera taken from patients early after embryo transfer have the potential to modulate the activation state of monocytes, using sera taken before embryo transfer as controls. As an index of monocyte activity we measured superoxide-dependent lucigenin-amplified chemiluminescence (Allen et al., 1985).

\section{Materials and methods}

The study population comprised 13 patients in whom pregnancy developed (ET +) after in-vitro fertilization and embryo transfer, performed as described previously (Sterzik et al., 1988). Twelve other patients, in whom no pregnancy developed after the same procedure performed at the same institution and in the same time period (ET -), were also studied.

Sera from patients were collected before and within 18 days after the procedure, sterile filtered and stored at $-20^{\circ} \mathrm{C}$ until assayed.

Mononuclear cells (MNC) were prepared from peripheral blood of healthy male volunteers by Ficoll gradient centrifugation (specific gravity of Ficoll $=1.077$ ) and washed twice in Hank's balanced salt solution without divalent cations. Finally, MNC were suspended at a density of $5 \times 10^{6} / \mathrm{ml}$ in phenol red-free minimal essential medium (T038, Biochrom/Seromed, Berlin, FRG) buffered to pH 7.2 with $20 \mathrm{mM} \mathrm{HEPES} \mathrm{(Calbiochem,} \mathrm{La}$ Jolla, USA) containing $100 \mu \mathrm{g} / \mathrm{ml}$ of bovine serum albumin (Behringwerke, Marburg, FRG) (T038/BSA). Flow-cytometric determination of cellular composition of $\mathrm{MNC}$ was done on an Ortho $50 \mathrm{H}$ cytofluorograph, using the following FITC-labelled murine monoclonal antibodies (Becton Dickinson Monoclonal Center, Mountain View, CA): anti-Leu 4 (T cells, CD3), antiLeu 12 (B cells, CD19), anti-LeuM3 (monocytes/macrophages) 
and anti-Leu 11 (CD16 on natural killer cells and granulocytes). The cellular composition of MNC from donor I (II) thus determined was the following: $75(82) \% \mathrm{~T}$ cells, $6(7) \%$ B cells, $15(10) \%$ monocytes, $5(4) \%$ NK cells, $<1(<1) \%$ granulocytes.

Mononuclear cell incubation with patient sera was performed as follows: $100 \mu \mathrm{l}$ aliquots of the MNC suspension $(=0.5 \times$ $10^{6} \mathrm{MNC}$ ) were dispensed in microtitre plate wells (Black microtitre plates, Dynatech, Embrach-Embraport, Switzerland) and incubated with $100 \mu \mathrm{l}$ of patient serum for $60 \mathrm{~min}$ at $37^{\circ} \mathrm{C}$. Thereafter, serum was removed by three cycles of centrifugation, aspiration of supernatant with a modified ELISA washer and renewed addition of T038/BSA medium, yielding a final serum concentration $<1 \%$. Control experiments showed that no cells were lost by this procedure.

Measurement of chemiluminescence (CL) from samples contained in microtitre plates was performed as follows. Cells were stimulated with $20 \mathrm{ng} / \mathrm{ml}$ of phorbol myristyl acetate in the presence of $100 \mu \mathrm{M}$ lucigenin ( $N, N$-dimethyl-acridinium nitrate; both from Sigma, München, FRG). The 60 min integral of CL was then measured using a computer-aided microtitre plate luminometer as described in more detail elsewhere (Maly $e t$ al., 1988). It was thus possible to complete the evaluation of all serum samples in triplicates on MNC of one donor within $<3 \mathrm{~h}$.

Because sera from different individuals affected the CL of test MNC differently, for each patient, the CL value obtained with her serum taken on the transfer day was used as reference and set at 100 . Results obtained with the other sera from the individual patient were expressed relative to this sample, yielding relative $\mathrm{CL}$ values in per cent.

To further compare the time course of relative $\mathrm{CL}$ values between the group of patients with successfully established pregnancy $(\mathrm{ET}+)$ and those in whom no pregnancy developed (ET -), the values of 3-day intervals before and after the day of embryo transfer where grouped together and averaged. Though this procedure diminished the temporal resolution of the study, it was necessary because for technical reasons (outpatient procedure) it was not possible to obtain a serum sample every day from each patient.

\section{Results and discussion}

Post-transfer sera from the majority of ET - patients enhanced the chemiluminescence response of male mononuclear cells to PMA, while post-transfer sera of ET + patients had only slight stimulatory or inhibitory effects (Figure 1). On average, this stimulatory activity of ET - sera appeared between days 4 and 6, was maximal between days 7 and 9 post-transfer (maximal value: $172 \pm 32 \%$, mean $\pm \mathrm{SEM}, N=9$ ) and thereafter returned to baseline levels. In contrast, the group of ET + patients showed on average no significant effects of post-transfer sera on chemiluminescence of indicator MNC (Figure 2). Differences between the relative CL means of ET - and ET + groups were statistically significant at days 4 to 6 and 7 to $9(P=0.0063$ for days $4-6$ and $P=0.065$ for days 7-9, Test 1 , Student's $t$-test). Interestingly, in six of eight individual ET - patients from whom sera in the period between days 4 and 6 were available, relative $C L$ values greater than the mean $+2 S D$ of the values of the ET+ group in this time interval were found. As can also
Rel. CL (\%)
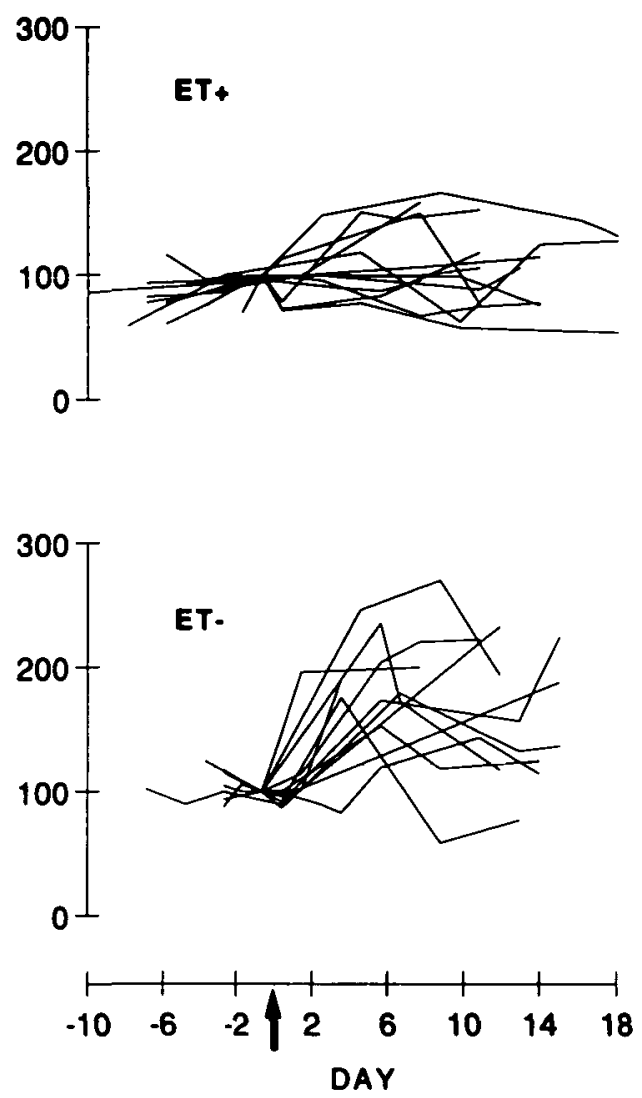

Fig. 1. Time course of MNC CL-modulating activity of individual sera from ET + (upper panel) and ET - patients (lower panel). For each patient, MNC chemiluminescence values are expressed as a percentage of the value obtained with her serum taken on or shortly before the day of embryo transfer, denoted as day 0 (arrow) and are linked by solid lines. Data are means of triplicate determinations with SD $<20 \%$ of the respective mean (Test 1).

be seen from Figure 2, these findings could be reproduced with target MNC from another donor.

The major cell type among blood mononuclear cells capable of producing $\mathrm{CL}$ was the monocyte, though a few contaminating granulocytes were present $(<1 \%)$. This makes likely the possibility that monocytes are the primary target cells of the MNC-CL enhancing activity described (Babior, 1978). However, it is also possible that the effect results from an interaction between serum factors, monocytes and other cells present in MNC, for example $T$ lymphocytes or natural killer cells, which may modulate the monocyte oxidative burst. Therefore, participation of NADPH oxidase-negative cells, such as T or NK cells, in the mechanism leading to the observed enhancement of mononuclear cell oxidative activity is to be further investigated.

Several soluble mediators are known to enhance the superoxideforming capacity of monocytes/macrophages, e.g. the lymphokines gamma-interferon and granulocyte-macrophage colony stimulating factor. However, they are mainly produced and consumed locally and generally not found in the circulation, and it is therefore unlikely that they could be responsible for the observed phenomenon. Further research is needed to examine the nature of the factor(s) responsible for the upward modulation 
ET POS TEST 1 ET NEG TEST 1
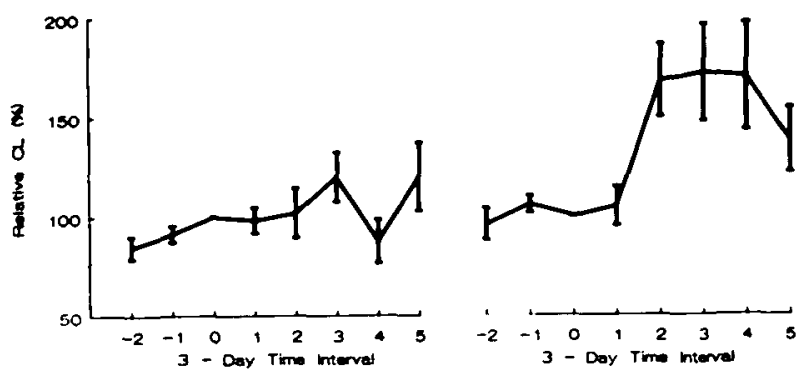

ET POS TEST 2

ET NEG TEST 2
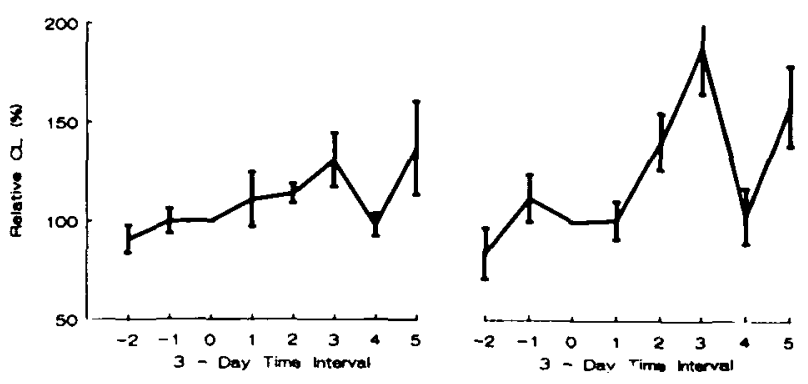

Fig. 2. Time course of mean MNC CL modulating serum activity. MNC CL modulation values obtained with MNC from two different donors (Test 1 and 2) were averaged over 3-day periods as described in Materials and methods. The day of embryo transfer is denoted day 0 . Data shown are mean \pm SEM. $P$ values indicate the significance of differences between ET + and ET-.

\section{of MNC CL.}

We had originally expected that sera from patients developing a pregnancy would exen inhibitory effects, in analogy with the findings of Morton et al. (1976) concerning EPF. We chose the phorbol ester PMA as trigger of the mononuclear cell oxidative burst because it is one of the most potent activators of the oxidative burst which also bypasses cell surface receptors and activates cell functions more directly, typically via protein kinase $\mathrm{C}$. Therefore, effects on PMA-induced responses, like the enhancement described, are likely to be caused at the postreceptor level, e.g. within the protein kinase $\mathrm{C}$-initiated signalling pathway or at the level of the NADPH oxidase itself. From this, it is probable that ET-sera will cause an enhancement of the MNC oxidative burst also when PKC and NADPH oxidase are activated via receptor-mediated mechanisms such as phagocytosis, though this will have to be tested experimentally.

Earlier, changes in the rosette-inhibitory activity of early pregnancy sera were reported which correlated with the outcome of mating or embryo transfer (early pregnancy factor; Morton et al., 1976). The finding that no differences in the MNC-CL modulatory activity of pre- and post-transfer sera of patients who developed a pregnancy were detected suggests that early pregnancy factor does not modulate monocyte oxidative burst, at least not in the amounts present in such sera. However, the finding of serum factors enhancing the monocyte oxidative burst in the group of patients in whom embryo transfer was not followed by establishment of pregnancy-originally devised as the control group-was unexpected and merits further investigation. The time at which these serum effects were noted would qualify them as associated with the late preimplantation or the peri-implantation period.

Elevated oestradiol levels and hence increased oestradiol/progesterone levels have been found to be associated with failed IVF attempts. However, no difference in oestradiol/progesterone ratios was evident between the ET + and ET - patient groups investigated in this study (data not shown). In addition, oestradiol and progesterone have been demonstrated to inhibit the superoxide-generating NADPH oxidase in vitro (Buyon et al., 1984). It is therefore unlikely that either of them could, alone, be responsible for the observed enhancement of superoxide generation.

In summary, the phenomenon we describe in this communication could be connected with the process itself which causes loss of the embryo. Thus, our data could be interpreted as further evidence for the existence of peripherally detectable parameters reflecting the fate of the embryo. Alternatively, the upward modulation of MNC CL in the patients that did not become pregnant, might merely be a consequence of the failure of pregnancy, such as, for instance, resolution of the corpus luteum. A study with serum samples taken throughout the menstrual cycle of normal women not attempting to conceive and taking no contraceptive medication could provide pertinent information; but due to obvious difficulties in obtaining such serum samples, these data are not yet available. Though it is therefore presently not possible to conclude which interpretation is correct, our data show that failure of an embryo to establish a pregnancy is associated with peripherally detectable factors effecting changes in immune cell function.

\section{References}

Allen,R.C., Mead,M.E. and Kelly,J.L. (1985) In Greenwald,R.A. (ed.), CRC Handbook of Methods for Oxygen Radical Research. CRC, Boca Raton, FL, pp. 343-351.

Babior,B.M. (1978) Oxygen-dependent microbial killing by phagocytes. N. Engl. J. Med., 298, 659-669.

Buyon,J.P., Korchak,H.M., Rutherford,L.E., Ganguly,M. and Weissmann,G. (1984) Female hormones reduce neutrophil responsiveness in vitro. Arthritis Rheum., 27, 623-630.

Imakawa,K., Anthony,R.V., Kazemi,M., Marotti,K.R., Polites,H.G. and Roberts,R.M. (1987) Interferon-like sequence of ovine trophoblast protein secreted by embryonic trophectoderm. Nature, 330, 377-379.

Maly,F.-E., Urwyler,A., Rolli,H.P., Dahinden,C.A. and de Weck,A.L. (1988) A single-photon imaging system for simultaneous quantitation of luminescent emissions from multiple samples. Anal. Biochem., 168, $462-469$.

Morton,H., Hegh,V. and Clunie,G.J.A. (1976) Studies of the rosette inhibition test in pregnant mice: evidence for immunosuppression? Proc. R. Soc. Lond. B, 193, 413-419.

O'Neill,C.O. (1985) Thrombocytopenia is an initial maternal response to fertilization in mice. J. Reprod. Feril., 73, 559-566.

Sterzik,K., Dallenbach,C., Schneider,V., Sasse, V. and DallenbachHellweg,G. (1988) In-vitro fertilization: the degree of endometrial insufficiency varies with the type of ovarian stimulation. Fertil. Steril., 50, 457.

Received on October 13. 1989: accepted on January 9, 1990 